\title{
DYNAMICS OF ASSEMBLED STRUCTURES: TAKING INTO ACCOUNT THE SURFACE DEFECTS IN INTERFACES
}

\author{
Nicolas PEYRET \\ SUPMECA PARIS \\ Saint-Ouen, FRANCE
}

\author{
Gaël CHEVALLIER \\ SUPMECA PARIS \\ Saint-Ouen, FRANCE
}

\author{
Jean-Luc DION \\ SUPMECA PARIS \\ Saint-Ouen, FRANCE
}

\section{ABSTRACT}

In structural dynamics, the prediction of damping remains the biggest challenge. This paper deals with the energy losses caused by micro-slip in a nominally planar interface of a structure. This paper proposes an analytical and experimental study of flexural vibrations of a clamped-clamped beam with innovative position of the interfaces.

The objective of this test bench is to characterize the global rheology of the interface. The proposed model aims to characterize this rheology based on local settings of the interface.

First, the test bench is described and the choice of the position of the interface is justified. The experimental bench and the dynamic behavior of this structure are presented. We propose to illustrate the mechanism of energy losses by micro-slip by making a comparison between the behavior of a "monolithic" beam and a sectioned beam.

Secondly, a modeling of the interface taking into account the surface defect is presented. The energy dissipated by friction in the interface is calculated during a loading cycle. This leads to a computation of the dissipated energy and thus to a nonlinear loss factor.

Finally, we confront the loss factor calculated analytically and the measured one.

\section{NOMENCLATURE}

\section{Symbol Description}

a Radius of circular contact

Units

c Radius of the sticking zone

df Height of the sphere

$\mathrm{E}^{*} \quad$ Composite modulus of elasticity

$\mathrm{G}^{*} \quad$ Inverse composite shear modulus

$\mathrm{h}$
Height difference between the two $\mathrm{m}$ types of spheres

$\begin{array}{lll}\mathrm{K}_{\mathrm{mcis}} & \text { Shear modal stiffness } & \mathrm{N} / \mathrm{m} \\ \mathrm{K}_{\mathrm{m}} & \text { Modal Stiffness } & \mathrm{N} / \mathrm{m} \\ \mathrm{N} & \text { Normal load } & \mathrm{N} \\ \mathrm{R} & \text { Radius of curvature of the sphere } & \mathrm{m} \\ \mathrm{T} & \text { Tangential load } & \mathrm{N} \\ \mathrm{W}_{\mathrm{diss}} & \text { Energy dissipation } & \mathrm{J} \\ \mathrm{W}_{\mathrm{ext}} & \text { External works } & \mathrm{J} \\ & & \\ \delta & \text { Tangential displacement } & \mathrm{m} \\ \delta_{\mathrm{ilim}} & \text { Limit of partial sliding } & \mathrm{m} \\ \delta_{\mathrm{n}} & \text { Normal approach } & \mathrm{m} \\ \xi & \text { Damping } & \% \\ \eta & \text { Loss factor } & \% \\ \mu & \text { Coefficient of friction } & \\ \rho & \text { Composite radius of curvature } & \mathrm{m} \\ v & \text { Displacement } & \mathrm{m} \\ \nu & \text { Poisson's ratio } & \mathrm{m} \\ \varnothing & \text { Diameter of the apparent surface } & \end{array}$

\section{INTRODUCTION - PRESENTATION OF THE TEST BENCH}

The present work starts from the idea of a previous paper [1]. Firstly the shape of the beam has been improved in order to obtain the greatest damping as possible by increasing the dimensions of the interfaces, see Peyret et al. [2]. The final design of this new benchmark was presented in Dion et al. [3]. The shape has been designed to avoid coupling between the normal stresses and the vibration motion even if the interfaces have been inaccurately located (Figure 1). 


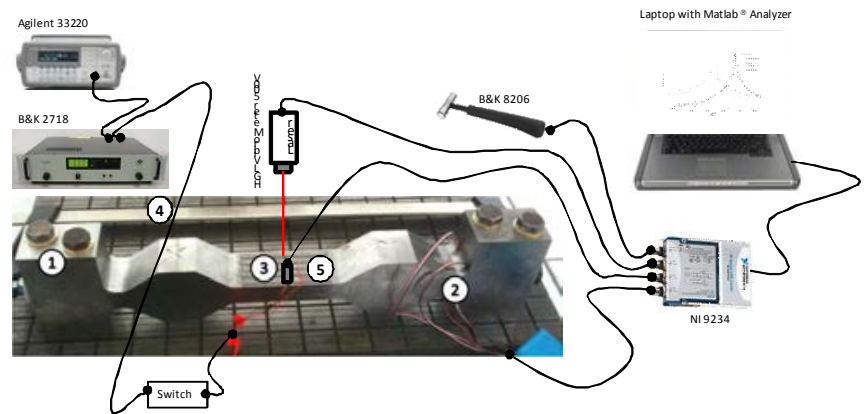

Figure 1. View of the benchmark screwed on its Steel Surface Plate. Part 1 is a massive part designed to clamp the beam on the heavy Steel Surface Plate. Parts 2 are the strain gauges glued on both sides of the beam to measure the normal load. Part 3 are the piezoelectric patches glued on both sides of the beam to excite the first vibration mode. Part 4 is a quick clamp used to apply the normal load before the tests. Part 5 is the Bruel \& Kjaer 4517 miniature accelerometer.

Piezoelectric transducers have been glued in order to excite the first vibration mode; sensors allow to obtain the normal load in the interfaces and to measure the transverse motion. An original experimental procedure is based on stop-sine excitation. The authors justify that their excitation method is more efficient that Dirac (shock) or Heaviside (release of a static load) excitations. Signal processing tools are proposed to post-process the instantaneous damping and frequency of the first mode of the structure. Finally this work experimentally demonstrates the presence of micro-slip damping, by comparing the vibration behavior of a monolithic part and a built-up structure with friction-joints (Figure 2). This comparison is the best way to determine the "added damping" [4].

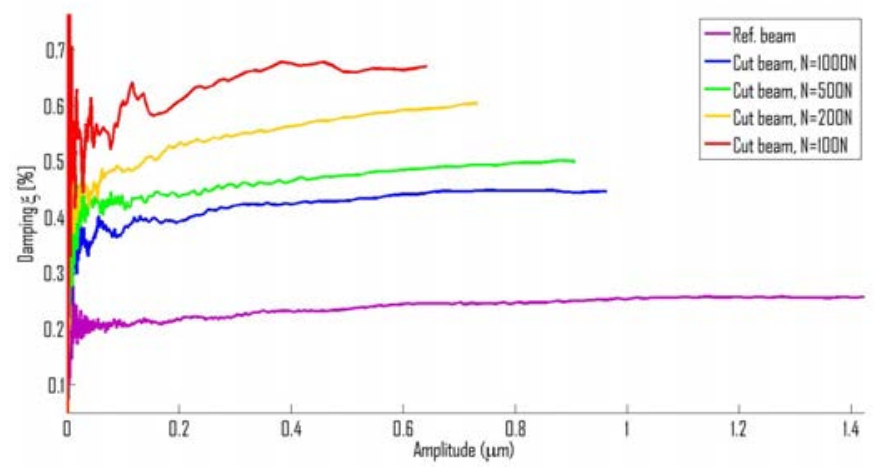

Figure 2. Evolutions of damping versus the normal load and versus the displacement magnitude. Both the monolithic (ref. beam) and the cut beam were tested. The latter was tested under four normal load levels in the range 100-1000 N

\section{MODELING OF THE INTERFACE: MULTIPLE SPHERICAL CONTACT}

When conducting functional surfaces (geometric area) specified by the design, it appears, in manufacturing, some geometrical defects. These defects are questioning the assumption of a plane contact interfaces in a uniform normal stress distribution.

The modeling of the interface by a plane surface can not take account the fact that there are energy losses for smallest oscillations (Figure 3), this is due to the surface defect.

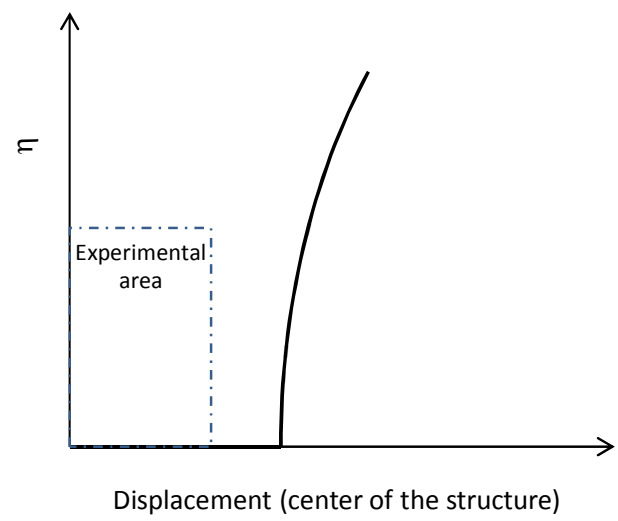

Figure 3. Loss factor $\eta(\%)$ vs $v(m)$ displacement at the center of the structure for plane surface.

To take into account the geometrical defects with contacts on several sphere, the contact interface is modeled as follows: contact is made with two types of sphere (Figure 4):

- $m$ spheres "G" ensuring the rigidity of the interface. They are the first to come into contact during the implementation of the normal load and can not slide completely (macro-slip) ;

- $n$ spheres "P" potentially slide completely.

Interface load is defined by the normal approach $\delta_{n}$ of the two normal planes constituting the interface. Before loading, the difference between heights of spheres is defined by $h$. The spheres are defined by their radius of curvature $\rho_{G}$ et $\rho_{P}$. 
Forces distribution on the spheres

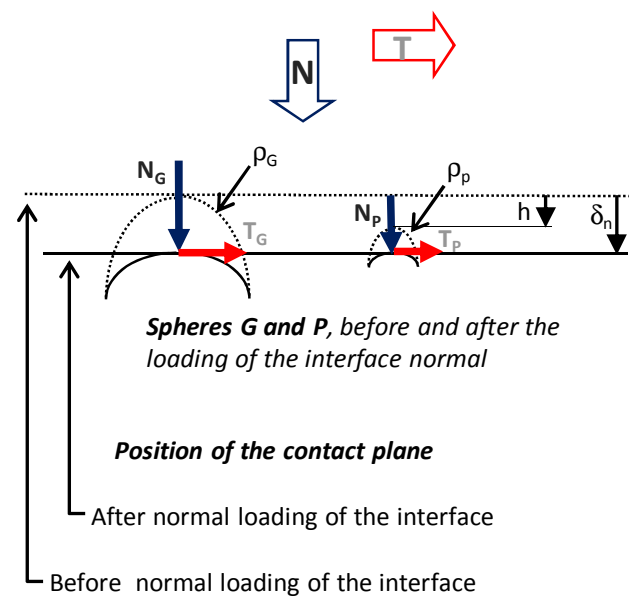

Figure 4. Modeling of the interface.

The distribution of forces on the interface is defined by: normal load $\mathrm{N}$ and tangential load $\mathrm{T}$. The distribution of these forces on different spheres constituting the contact by $\mathrm{N}_{\mathrm{i}}$ and $\mathrm{T}_{\mathrm{i}}$.

$$
\begin{aligned}
& N=m N_{G}+n N_{P} \\
& T=m T_{G}+n T_{P}
\end{aligned}
$$

The distribution of the normal load is a function of the normal approach $\delta_{\mathrm{n}}$ and is defined, for one spherical contact, by the Hertz theory [4]:

$$
\delta_{n}=\frac{1}{\rho}\left(\frac{3}{4} \cdot \frac{N_{i} \cdot \rho}{E^{*}}\right)^{2 / 3}
$$

With

$$
\frac{1}{E^{*}}=\frac{1-v_{1}^{2}}{E_{1}}+\frac{1-v_{2}^{2}}{E_{2}} \quad \frac{1}{\rho}=\frac{1}{R_{1}}+\frac{1}{R_{2}}
$$

Using the forces distribution given on figure 4, the normal load on the interface can be expressed by:

$$
N=\frac{4}{3} E *\left(m \rho_{G}^{\frac{1}{2}} \delta_{n}^{\frac{3}{2}}+n \rho_{P}^{\frac{1}{2}}\left(\delta_{n}-h\right)^{\frac{3}{2}}\right)
$$

Once the normal load is applied, a tangential displacement $\delta$ is applied between both surfaces of the interface. The assumption is that this displacement is the same for each contact.

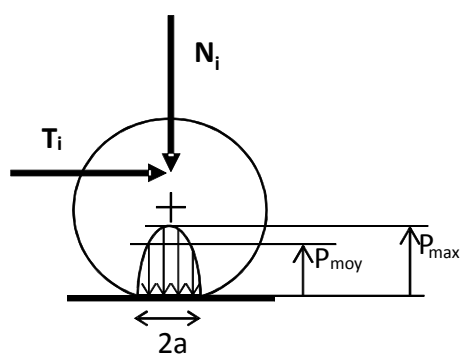

Contact area

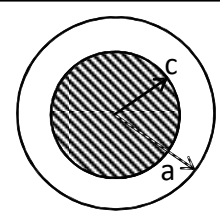

Stick zone Slip zone

Figure 5. Schematic elastic spheres pressed by a constant normal force $N_{i}$ and subjected to an oscillating tangential force $T_{i}$.

The Mindlin theory [6-7], gives the displacement from the partial slip into the contact surface (defined by c on Figure 5):

$$
\delta=\frac{3 \mu N_{i}}{16} G^{*} \frac{\left(a^{2}-c^{2}\right)}{a^{3}}
$$

with

$$
G^{*}=\frac{2-v_{1}}{G_{1}}+\frac{2-v_{2}}{G_{2}} \text { and } G_{j}=\frac{E_{j}}{2\left(1+v_{j}\right)}
$$

From the previous equations, Johnson [8] offers solutions for a spherical contact, initially loaded with a constant normal load $\mathrm{Ni}$, and subjected to a tangential load $\mathrm{Ti}$ oscillating between $\pm \mathrm{T}_{\max }$ with $T_{\max }<\mu N_{i}$ (Figure 6).

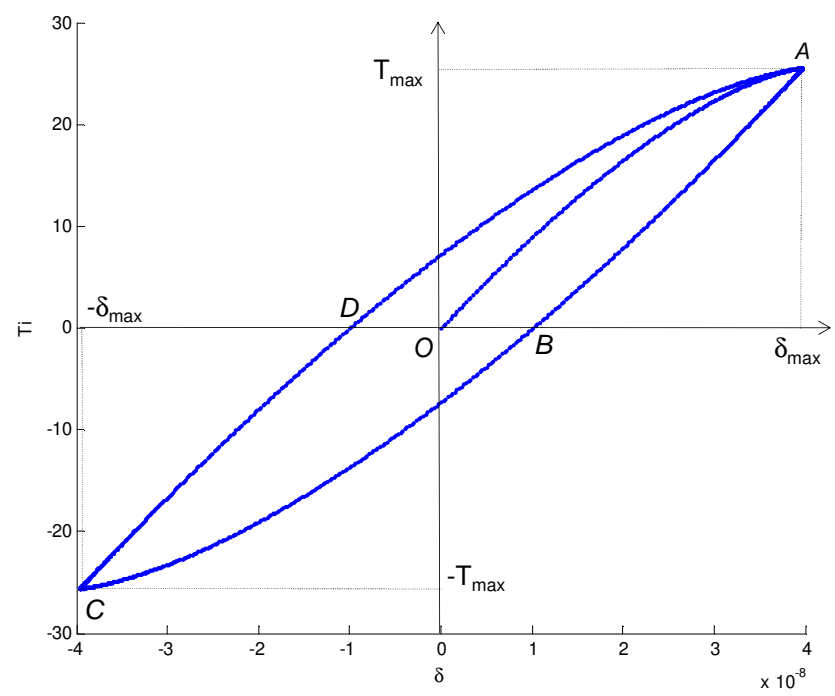

Figure 6. Loading cycle for one spherical contact. Tangential load $T i(N)$ vs $\delta$ displacement in the contact ( $\mathrm{mm}$ ). 
The surface defined by the cycle gives the dissipated energy for a cycle. This energy can be expressed as a function of the tangential displacement $\delta$ :

$$
W_{\text {diss }}=\frac{9 \mu^{2} N_{i}^{2}}{10 a_{i}} G^{*}\left[1-\left(1-\frac{16 a_{i}}{3 \mu N_{i} G^{*}} \delta\right)^{\frac{5}{2}}-\frac{5}{6}\left(1-\left(1-\frac{16 a_{i}}{3 \mu N_{i} G^{*}} \delta\right)^{\frac{3}{2}}\right)\left(2-\frac{16 a_{i}}{3 \mu N_{i} G^{*}} \delta\right)\right]
$$

From the Mindlin theory [6-7] $\delta_{\text {ilim }}$ is calculated for one tangential load. $\delta_{\text {ilim }}$ define a limit value of tangential displacement for each type of spherical contact: passage from macro-slip phase to total slip phase.

$$
\delta_{i \lim }=\frac{3 \mu N_{i}}{16 a_{i}} G^{*}
$$

This limit is defined on the loading cycle with macro-slip (Figure 7).

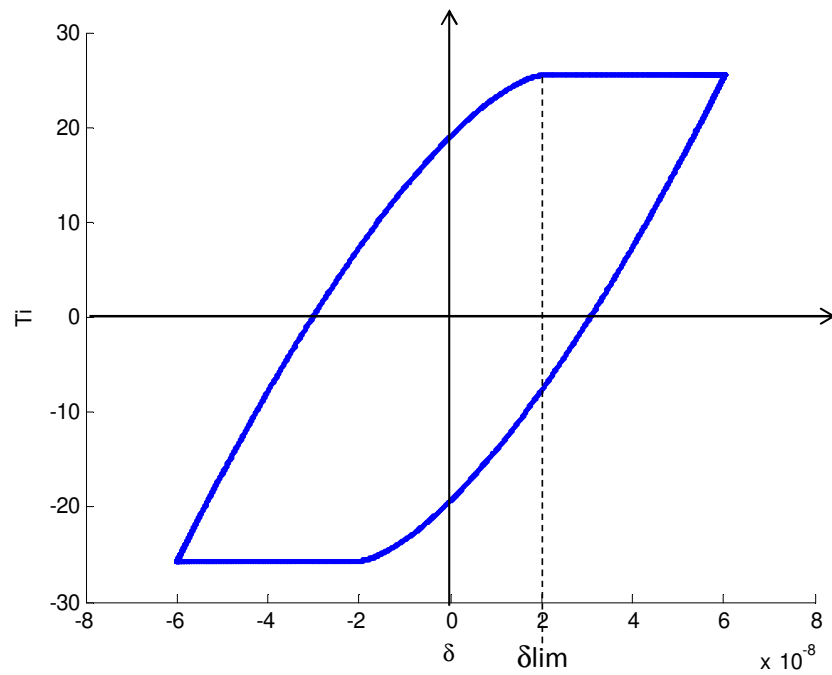

Figure 7. Loading cycle with macro-slip for one spherical contact. Tangential load $T i(N)$ vs $\delta$ displacement in the contact (mm).

In function of the value of $\delta$, two phases can be highlighted:

Spheres G and P did not slip totally: phase called "generalized partial slip" (GPS);

Spheres G remain in partial slip and spheres P slip totally: phase called "pseudo-partial slip" (PPS).

The distribution of the tangential load, is function of the normal approach $\delta_{\mathrm{n}}$ :
For the phase of generalized-partial slip (GPS)

$$
T=m \mu N_{G}\left[1-\left(1-\frac{16 a_{G}}{3 \mu N_{G} G^{*}} \delta\right)^{\frac{3}{2}}\right]+n \mu N_{P}\left[1-\left(1-\frac{16 a_{P}}{3 \mu N_{P} G^{*}} \delta\right)^{\frac{3}{2}}\right]
$$

For the phase of pseudo-partial slip (PPS)

$$
T=m \mu N_{G}\left[1-\left(1-\frac{16 a_{G}}{3 \mu N_{G} G^{*}} \delta\right)^{\frac{3}{2}}\right]+n \mu N_{P}
$$

The loading cycle of the multiple spherical contacts are plotted on figure 8 .

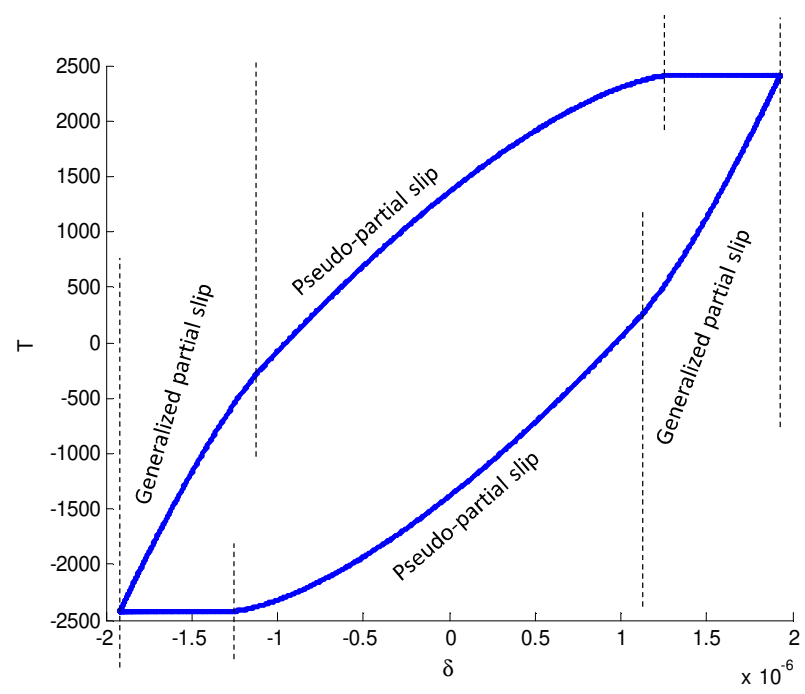

Figure 8. Tangential load $T i(N)$ vs diplacement $\delta(m)$

Comparing the shape of the cycle to that given by a single spherical contact (Figure 7) shows a new behavior during the loading especially when the spheres "P" begin to slip completely.

\section{CHARACTERIZATION OF THE JOINT SURFACES OF THE TEST BENCH}

To obtain the real contact surfaces of the interfaces some 3D measurements of these surfaces were made.

On each surface, a million points were measured. After filtering and re-sampling, we obtain a mesh of points on the surface of $1200 \times 800$ dots.

The contact being made on the more external spheres of the material, and so as to define a cloud of points defining possible areas of contact, it was chosen to retain only the "higher" points ( $10 \%$ of the set of points). 
Figure 9 shows that the surfaces are composed of several spherical zones on which contact will be established.

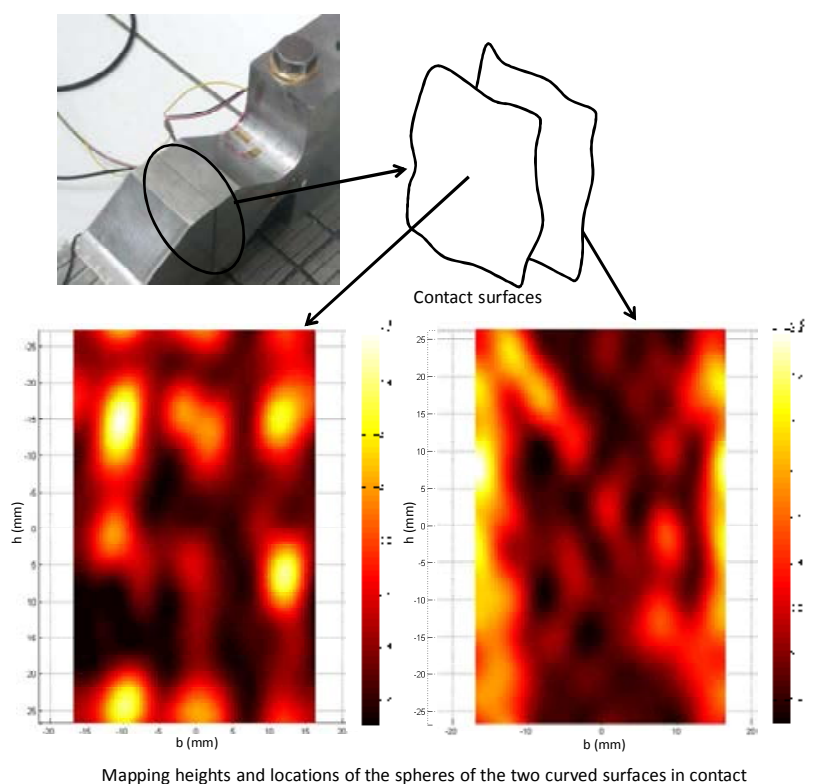

Figure 9. Measures three-dimensional surfaces of a testbench interfaces (measurements in $\mathrm{mm}$ ).

To determine the influence of defects of forms on the damping, the interface is modeled.

We model the spheres of the surfaces as follows:

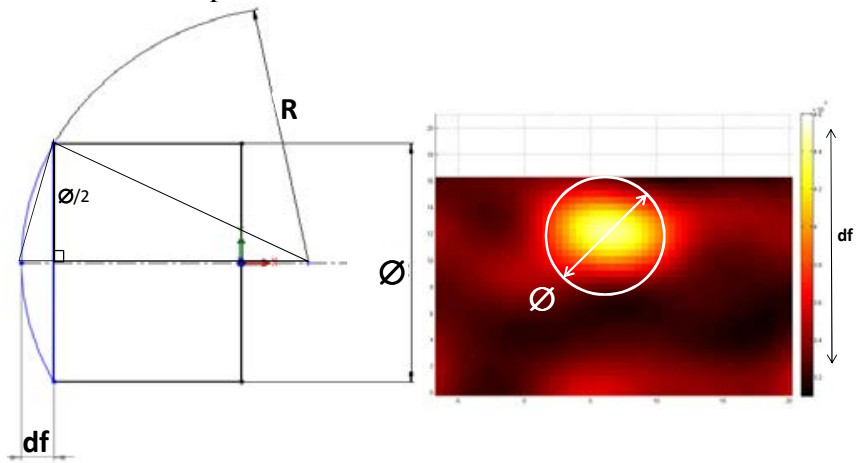

Figure 10. Geometric modeling of the sphere (left) Identification of the parameters of the sphere on the measurement (right).

With R: radius of curvature of the sphere, df: height of the sphere and $\varnothing$ the diameter of the apparent surface of the sphere.

The radius $\mathrm{R}$ is then calculated using the following expression:

$$
R=\frac{\left(\frac{\varnothing^{2}}{4}+d f^{2}\right)}{2 d f}
$$

Two types of sphere are identified in accordance with the model:

\begin{tabular}{|c|c|c|c|c|}
\hline Spheres & Number & $\mathrm{df}(\mathrm{m})$ & $\varnothing(\mathrm{m})$ & $\mathrm{R}(\mathrm{m})$ \\
\hline $\mathrm{G}$ & $\mathrm{n}=5$ & $1,70 \mathrm{E}-06$ & $1,80 \mathrm{E}-02$ & $2,38 \mathrm{E}+01$ \\
\hline $\mathrm{P}$ & $\mathrm{m}=18$ & $1,20 \mathrm{E}-06$ & $1,60 \mathrm{E}-02$ & $2,67 \mathrm{E}+01$ \\
\hline \multicolumn{5}{|c|}{$\mathrm{h}=2.4 \mathrm{E}-07 \mathrm{~m}$} \\
\hline
\end{tabular}

Table 1 : Model parameters

These two types of spheres exist on each surface. During the normal loading of the interface and considering the position of each sphere, it is considered that the spheres come in contact with a surface considered flat. The spheres $\mathrm{G}$ are the first and most compressed, they ensure the rigidity of the interface. The spheres $P$ are slightly compressed.

\section{LOSS FACTOR}

The equation defining the energy dissipated by one spherical contact (9) and (10), are used to define the energy dissipated by the multiple spherical contact:

For the phase of generalized partial slip (GPS)

$$
\begin{aligned}
& W_{\text {diss }}=\frac{9 \mu^{2}}{10} G^{*}\left\{m \frac{N_{G}{ }^{2}}{a_{G}}\left[1-\left(1-A_{G} \delta\right)^{\frac{5}{2}}-\frac{5}{6}\left(1-\left(1-A_{G} \delta\right)^{\frac{3}{2}}\right)\left(2-A_{G} \delta\right)\right] \ldots\right. \\
& \left.\ldots+n \frac{N_{P}{ }^{2}}{a_{P}}\left[1-\left(1-A_{P} \delta\right)^{\frac{5}{2}}-\frac{5}{6}\left(1-\left(1-A_{P} \delta\right)^{\frac{3}{2}}\right)\left(2-A_{P} \delta\right)\right]\right\} \\
& \text { avec } A_{i}=\frac{16 a_{i}}{3 \mu N_{i} G^{*}}
\end{aligned}
$$

For the phase of pseudo- partial slip (PPS)

$$
\begin{aligned}
& W_{\text {diss }}=\frac{9 \mu^{2}}{10} G^{*}\left\{m \frac{N_{G}{ }^{2}}{a_{G}}\left[1-\left(1-A_{G} \delta\right)^{\frac{5}{2}}-\frac{5}{6}\left[1-\left(1-A_{G} \delta\right)^{\frac{3}{2}}\right)\left(2-A_{G} \delta\right)\right]+n \mu N_{P} \delta\right\} \\
& \text { avec } A_{i}=\frac{16 a_{i}}{3 \mu N_{i} G^{*}}
\end{aligned}
$$

The energy dissipated is plotted on figure 11 .

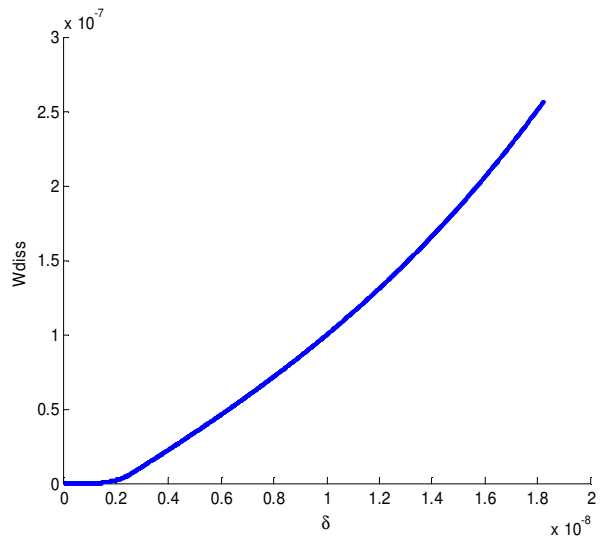

Figure 11. Energy dissipated $W_{\text {diss }}(\mathrm{J})$ vs diplacement $\delta(\mathrm{m})$ 
Taking into account the external force applied on the structure to obtain the dynamic behavior, it is possible to define the loss factor of the structure:

$$
\eta=\frac{W_{\text {diss }}}{2 \pi W_{e x t}}
$$

The energy dissipated by the load cycle $\mathrm{W}_{\text {diss }}$ is defined by sections (equations (12) and (13)) depending on the value of the tangential displacement $\delta$ and $\delta_{\text {ilim }}$ (equation (8))

The work of external forces $\mathrm{W}_{\text {ext }}$ can be expressed in terms of displacement at the center of the beam $v$ :

$$
W_{e x t}=\frac{1}{2} K_{m} \cdot v^{2}
$$

with $\mathrm{K}_{\mathrm{m}}=2.8110^{7} \mathrm{~N} / \mathrm{m}$ : the modal stiffness of the structure. This gives the work of external forces applied to the structure:

$$
W_{e x t}=\frac{1}{2} K_{m} \cdot\left(\frac{T_{\max }}{K_{m c i s}}\right)^{2}
$$

with $\mathrm{K}_{\text {mcis }}=3.2310^{7} \mathrm{~N} / \mathrm{m}$ : the shear modal stiffness of the structure.

We can plot the loss factor evolution of the structure for a normal pre-load of $460 \mathrm{~N}$ on the interface (Figure 12). The transition from GPS to PPS leads a drastic change in the evolution of the loss factor.

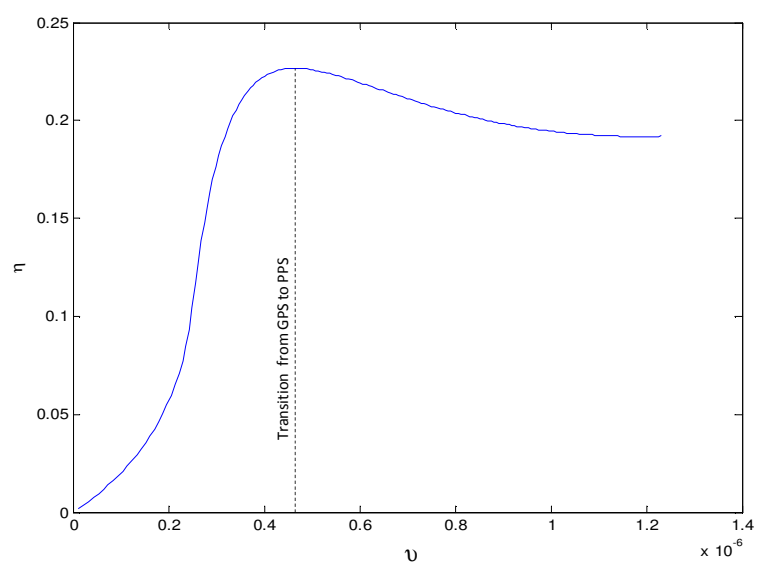

Figure 12. Loss factor $\eta(\%)$ vs $v(m)$ diplacement at the center of the structure for one multiple spherical contact surface.

To compare the experimental results with the results provided by the model, we need to isolate the contribution of interfaces studied of the contribution of the other sources of damping. For this part the experimental results were linearized by parts.

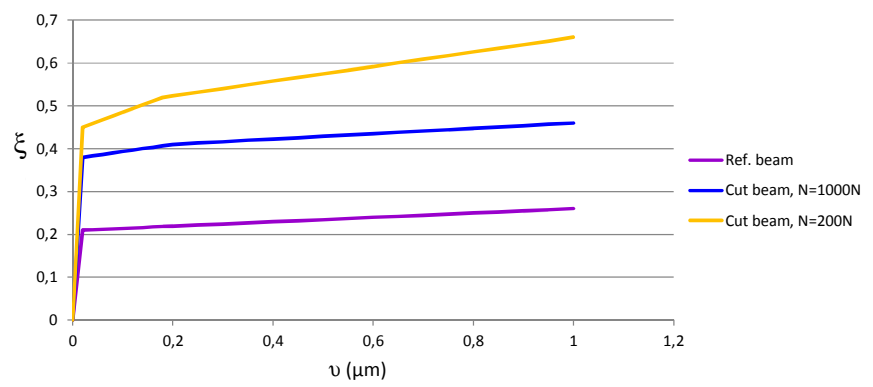

Figure 13. Linearized experimental damping $\xi(\%)$ vs $v(m)$.

The curves in Figure 13 show the evolution of the damping of the monolithic structure (ref. beam) and of the assembled structure in the case of a normal load of $1000 \mathrm{~N}$ and $200 \mathrm{~N}$. The two structures, assembly and monolithic, have the same geometry and the same boundary conditions, it is thus possible to consider two distinct sources of damping. We distinguish the dissipation induced by the interfaces $f_{\mathrm{d} 2}$ of the dissipation provided by all the others sources $\mathrm{f}_{\mathrm{d} 1}$ (clamps, intrinsic damping of the material ...):

$$
m \ddot{x}+k x=f_{d 1}(x, \dot{x})+f_{d 2}(x, \dot{x})
$$

The dissipated energy can be written as:

$$
W_{\text {diss }}=\oint\left(f_{d 1}(x, \dot{x})+f_{d 2}(x, \dot{x})\right) d x
$$

Both energies are separable by subtraction. It is considered that $\mathrm{W}_{\mathrm{d} 1}$ can be identified by a test on the monolithic beam and that $\mathrm{W}_{\mathrm{d} 2}$ can be calculated by subtracting the damping rate of the monolithic beam to those of the beam assembled. Thus the contribution of the two interfaces is isolated in the damping of the structure (Figure 14).

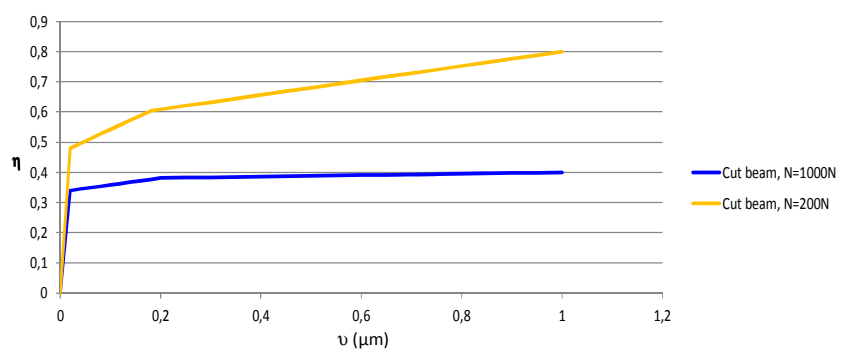

Figure 14. Loss factor $\eta(\%)$ vs $v(m)$ diplacement at the center of the structure for multiple spherical contact surface.

We can compare these experimental results with those given by the model (Figure 15). We note that we reached the same levels of damping, although this level is reached experimentally for more smallest oscillations. This difference is probably due to the choice of a contact model at two types of spheres of different heights. Indeed this model does not take into account the progressivity of the spheres height in the interfaces and the 
fact that probably some irregularity of the surface outcrop the contact and dissipate some energy from smallest amplitudes of vibration.

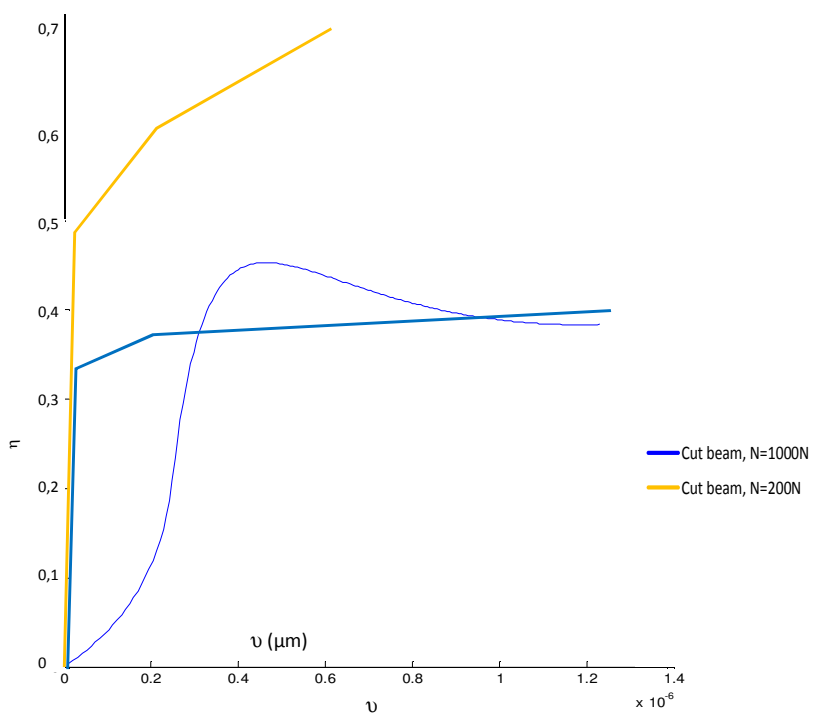

Figure 15. Loss factor $\eta(\%)$ vs $v(m)$ diplacement at the center of the structure, comparaison of experimental and model results.

\section{CONCLUSION}

Unlike a modeling that takes into account the interface as a plan geometrically perfect [1], taking into account the surface defects it is possible to model the presence of damping in the interfaces as soon the smaller amplitudes of vibration.

A model that takes into account surface defects was developed to more accurately model the evolution of the damping as a function of the amplitude of the deformations. This new approach is a multi-spherical modeling of the interfaces, based on the behavior of the spherical contact developed by Hertz and Mindlin.

This modeling allows us to compare the loss factor found experimentally

\section{REFERENCES}

[1] N. Peyret, J.L. Dion, G. Chevallier, and P. Argoul. Micro slip Induced damping in planar contact under constant and uniform normal stress. International Journal of Applied Mechanics. 2(2), 281-304, 2010.

[2] N. Peyret, J.L. Dion, G. Chevallier, and P. Argoul.P. Non linear dynamic behavior modelling of a planar friction interface in a structure assembly. In Proc. IDETC ASME, 2009

[3] J.L. Dion, G. Chevallier, and N. Peyret. Improvement of the measurement techniques for the damping induced by micro sliding. Mech. Syst. Signal Process, v. 34, p. 106-115, 2013.

[4] H. Hertz. On the contact of elastic solids. J. Reine Angew. Math., vol. 92, p156-171, 1882.

[5] D.J. Segalman. A modal approach to modeling spatially distributed vibration energy dissipation. Sandia Report , 2010.

[6] R.D. Mindlin. Compliance of elastic bodies in contact. ASME J. Appl. Mech, v. 16, p. 259-268, 1949.

[7] R.D. Mindlin. Effects of an oscillating tangential force on the contact surfaces of elastic spheres. Proc. 1st US National Congress of Applied Mechanics, ASME, New York, p. 203, 1952.

[8] K.L. Johnson. Contact Mechanics. Cambridge University Press, London, 1985 